Keywords: Sleep disorders; Polysomnography; Obstructive sleep apnoea.

\title{
Sleep disordered breathing in community psychiatric patients
}

\author{
Kirstie N. Anderson* \\ Tony Waton** \\ Daniel Armstrong ** \\ Helen M Watkinson** \\ Paul Mackin** \\ * Department of Neurology, Royal Victoria \\ Infirmary, Newcastle upon Tyne \\ ** Academic Psychiatry, Newcastle General \\ Hospital, Newcastle upon Tyne \\ UNITED KINGDOM
}

\begin{abstract}
Background and Objectives: Sleep disturbance is prominent in many neuropsychiatric disorders and may precipitate or exacerbate a range of psychiatric conditions. Few studies have investigated sleep disordered breathing and in particular obstructive sleep apnoea in community psychiatric patients and the commonly used screening instruments have not been evaluated in patients with psychiatric disorders. The objective is to evaluate the prevalence of sleep disordered breathing in a community cohort with chronic mental illness on long term psychotropic medication, and to assess the effectiveness of commonly used screening instruments to detect abnormal sleep.

Methods: 52 patients completed sleep questionnaires and 50 undertook overnight oximetry.

Results: 52\% ( $\mathrm{n}=26)$ had sleep-disordered breathing; 20\% $(\mathrm{n}=10)$ had moderate/severe sleep apnoea. The Epworth Sleepiness Score and the Pittsburgh Sleep Quality Inventory did not predict sleep disordered breathing.

Conclusions: Patients with psychiatric disorders in the community have a high rate of undiagnosed sleep disordered breathing, which is not reliably detected by established sleep disorder screening questionnaires.
\end{abstract}

Received: 5 May 2011

Accepted: 28 November 2011 


\section{Introduction}

Sleep disturbance is prominent in many neuropsychiatric disorders and disturbed sleep is found in $30-80 \%$ of patients with schizophrenia and $90 \%$ of those with major affective disorders ${ }^{1}$. Poor subjective sleep quality is associated with reduced quality of life ${ }^{2}$.

Traditionally sleep disturbance is regarded as secondary to the mental illness but there is growing evidence that primary sleep disorders such as obstructive sleep apnoea can trigger or exacerbate a range of psychiatric conditions.

Sleep disordered breathing is common and is caused by pauses in breathing (apnoea) or abnormally shallow breathing (hypopnoea). Obstructive sleep apnoea (OSA) is seen with snoring and is estimated to affect $4 \%$ of men and $2 \%$ of women aged 30 $60^{3}$. The consequences are disrupted night sleep and subsequent daytime sleepiness, there is an effective treatment for many symptomatic patients with the use of continuous positive airway pressure. The current diagnostic criteria require overnight oximetry showing $>5$ apnoeas per hour of sleep in association with either daytime sleepiness, fatigue, insomnia, nocturnal choking or reported loud snoring. In the absence of reported symptoms it can be diagnosed with > 15 apnoeas per hour (American Academy of Sleep Medicine, ICSD 2005). Patients with OSA have impaired cognition and an increased incidence of depression which improves following treatment ${ }^{4}$. There is a strong association with obesity and increasing evidence for an association with metabolic syndrome. Sleep disturbance in psychiatric populations may be due to the psychiatric illness, psychotropic medication or the result of a primary sleep disorder. The prevalence of sleep apnoea in the psychiatric population in the community has been studied with conflicting results ${ }^{5-7}$.
There is an increased prevalence of metabolic syndrome in those with chronic mental illness and a recent cross sectional study has reported the occurrence of metabolic syndrome in $33 \%$ of those with chronic mental illness on long term antipsychotic medication $^{8}$. There is accumulating evidence of an association between metabolic syndrome and obstructive sleep apnoea ${ }^{9}$. Whether metabolic syndrome is independently associated with obstructive sleep apnoea remains controversial and the association with insulin resistance has been studied with conflicting results ${ }^{10}$. There have been no cross sectional or prospective studies of psychiatric patients with metabolic syndrome to assess the prevalence of OSA. Previous in patient studies have shown that obese patients with chronic psychiatric morbidity have high rates of $\mathrm{OSA}^{11}$. Occasional case reports have identified patients whose psychiatric symptoms improved with treatment of their OSA ${ }^{12}$.

Several sleep questionnaires are used as clinical and research tools to screen for sleep disorders but their validity has not been established in patients with psychiatric disorders. Two of the commonest self report questionnaires of daytime sleepiness and sleep quality respectively are the Epworth Sleepiness Score ${ }^{13}$ and the Pittsburgh Sleep Quality Index ${ }^{14}$ but they have not been validated in patients with chronic mental health problems. Therefore there is little evidence at present for the best method of assessing those at risk of sleep apnoea within this population.

In this pilot study, we screened for sleep apnoea in community psychiatric patients. We investigated the relationship between abnormal sleep and metabolic features, and assessed the sensitivity and specificity of commonly used screening instruments for detecting sleep apnoea in this population. 


\section{Methods}

A cohort of patients within the North East of England has been identified and recruited from secondary care community psychiatric services. This cohort has been studied longitudinally at baseline, 18 months and 3 years and has undergone detailed metabolic assessments to determine the presence or absence of the metabolic syndrome. $52(57 \%)$ of the original 90 patients from this longitudinal cohort agreed to participate in this study. All patients were assessed between December 2008 and January 2010.

The only inclusion criteria were that patients were treated within the community and had an established mental illness. Exclusion criteria were as follows: patients who were already being treated for a known primary sleep disorder, patients with an eating disorder and/or patients with established end-stage major organ disease.

All patients completed an Epworth Sleepiness Score (ESS) questionnaire and the Pittsburgh Sleep Questionnaire Inventory (PSQI). The ESS has been validated as a screening questionnaire for obstructive sleep apnoea in the general population ${ }^{13}$. Demographic data, neck circumference, body mass index (BMI), blood pressure and current medications were recorded. A single overnight domiciliary partial polysomnographic assessment was performed with the Embletta X10 portable digital recorder ${ }^{15}$. Respiratory effort was detected with chest and abdominal bands measuring inductance, airflow was detected with nasal cannulae measuring pressure as well as the presence of snoring, and oxygen saturation of arterial blood was also measured in order to detect sleep apnoea syndrome. Heart rate was measured and an accelerometer recorded body position. All respiratory events were scored according to standard criteria of the American
Association of Sleep Medicine ${ }^{16}$. The apnoea hypopnoea index (AHI) and oxygen desaturation index (ODI) were derived. The apnoea hypopnoea index is the number of apnoeas or hypopnoeas in an hour and the oxygen desaturation index is the number of times the oxygen level drops $>4 \%$ of the baseline saturation in an hour. An AHI of $>$ $5 / \mathrm{hr}$ was considered abnormal and indicative of sleep apnoea. Severity was further defined as mild (AHI 5-15), moderate (AHI $15-30)$ or severe (AHI >30). Those with the obstructive sleep apnoea syndrome were defined as per the 2005 AASM criteria.

All patients gave their fully informed consent to participate in this study which was approved by the local Research Ethics Committee.

\section{Data analysis}

Data were analysed using the Statistical Package for the Social Sciences (SPSS) for Windows, version 17.0. Comparisons between groups were made using $\chi^{2}$ and Student's t-test, as appropriate. Bivariate correlation analysis was used to investigate the association between the apnoea-hypopnoea and oxygen desaturation indices and the continuous variables age, weight, BMI, neck circumference, blood pressure, $\%$ of BNF maximum dose of antipsychotic drug, total number of psychotropic drugs and ESS and PSQI scores. The sensitivity and specificity of the ESS and PSQI questionnaires for detecting sleep disordered breathing were calculated. Linear and binary logistic regression analyses were performed to identify independent predictors of AHI and AHI $>5$, respectively. All reported $\mathrm{p}$ values are 2 tailed. Statistical significance was defined as $\mathrm{p}<0.05$. 


\section{Results}

\section{Demographic data}

52 patients $($ female $=22$; male $=30$ ) underwent metabolic assessment, sleep questionnaires and overnight sleep studies. Two patients failed to tolerate the overnight sleep study, so data analysis was undertaken on the 50 remaining patients. The demographic data, diagnostic distribution and medication of the 52 patients studied are shown in table 1. The majority had an elevated BMI with $44(84 \%)>25 \mathrm{~kg} / \mathrm{m}^{2} ; 29$ patients $(56 \%)$ were classified as obese with a BMI 30 - 39 $\mathrm{kg} / \mathrm{m}^{2}$ and $2(4 \%)$ were morbidly obese with BMI $>40 \mathrm{~kg} / \mathrm{m}^{2} .22$ patients $(42 \%)$ were hypertensive at baseline assessment. Many of the patients studied either lived or slept alone so a witness history to corroborate the presence or absence of snoring or apnoeas could not be obtained.

Table 1

Demographics, diagnostic distribution and profile of psychotropic drugs

\begin{tabular}{lr}
\hline Age, years, mean $( \pm \mathrm{SD})$ & $50.2(10.7)$ \\
Gender, male:female, $\%(\mathrm{n})$ & $58: 42(30: 22)$ \\
$\mathrm{BMI}>30 \mathrm{~kg} / \mathrm{m}^{2}, \%(\mathrm{n})$ & $59.6(31)$ \\
Hypertensive, $\%(\mathrm{n})$ & $42.3(22)$ \\
\hline Diagnosis, \% (n) & $44.2(23)$ \\
Schizophrenia & $36.5(19)$ \\
Bipolar affective disorder & $3.8(2)$ \\
Schizoaffective disorder & $7.7(4)$ \\
Personality disorder & $5.8(3)$ \\
Depressive disorder & $1.9(1)$ \\
Delusional disorder & $80.8(42)$ \\
\hline Treatment with antipsychotic, $\%(\mathrm{n})$ & $7.7(4)$ \\
Above $100 \%$ BNF dose of antipsychotic, \% (n) & $42.3(22)$ \\
Treatment with antidepressant, \% (n) & $30.8(16)$ \\
Treatment with mood stabiliser, \% (n) & $21.2(11)$ \\
Treatment with benzodiazepine $\%,(\mathrm{n})$ & \\
\hline
\end{tabular}

BMI - body mass index; BNF - British National Formulary.

\section{Sleep questionnaire data}

52 patients $(100 \%)$ completed the ESS, and scores ranged between 0 and 24 . The mean ESS was $5.6(\mathrm{SD} \pm 5.04)$. The majority $(\mathrm{n}=44 ; 85 \%)$ had scores within the normal range of $<10$. Fifty patients (male $=28$, female $=22$ ) completed the PSQI with 28 $(56 \%)$ having subjective sleep disturbance with an abnormally elevated score $>5$. The mean PSQI was 6.5 (s.d. 3.5). Severe sleep disturbance (PSQI $>10$ ) was reported by 7 (13\%) patients. Bivariate correlation analysis revealed a significant correlation between ESS and PSQI global scores $(r=0.38$, $\mathrm{p}=0.007)$. The mean PSQI score was statistically significantly higher in those individuals with an abnormal (>5) ESS (9.4, s.d. 
3.4) compared to individuals with a normal $\operatorname{ESS}(5.9$, s.d. 3.3; $\mathrm{t}=-2.7$, df 48, $\mathrm{p}=0.009)$. The component scores that make up the overall PSQI score were analysed separately to see if any of the separate components correlated with either AHI, ODI or ESS but there was no significant association with any of the components.

\section{Sleep study data}

Sleep disordered breathing defined as an AHI $>5$ events per hour was present in 26 $(52 \%)$ patients $(32 \%$ mild, $14 \%$ moderate and $6 \%$ severe); see table 2 . Oxygen desaturation index (ODI) was available in 49 pa- tients and $12(23.1 \%)$ patients had an ODI of $>5 / \mathrm{hr}$ (males $=37 \%$, females $=9 \% ; \chi^{2}=$ 5.1 , df $1, \mathrm{p}=0.02)$. A higher percentage of men $(60.7 \%)$ had SDB compared to women $(36.4 \%)$, although this difference did not reach statistical significance $\left(\chi^{2}=2.9\right.$, df 1 , $\mathrm{p}=0.08)$; no women had severe sleep apnoea. The nasal flow was used to determine the presence or absence of snoring and 30 $(60 \%)$ patients snored during the sleep study although the percentage of time spent snoring was highly variable between patients. Respiratory events were further classified as obstructive or central apnoeas. Only $3(6 \%)$ patients had central apnoeas $>5$ per hour; the majority of events were obstructive.

Table 2.

Domiciliary polysomnography data on 50 patients

\begin{tabular}{lccc} 
& Total $(\mathrm{n}=50)$ & Male $(\mathrm{n}=28)$ & Female $(\mathrm{n}=22)$ \\
& $\%(\mathrm{n})$ & $\%(\mathrm{n})$ & $\%(\mathrm{n})$ \\
\hline AHI 0-5 (normal) & $48.0(24)$ & $35.7(10)$ & $63.6(14)$ \\
AHI 5-15 (mild) & $32.0(16)$ & $39.3(11)$ & $22.7(5)$ \\
AHI 15-30 (moderate) & $14.0(7)$ & $14.3(4)$ & $13.6(3)$ \\
AHI > 30 (severe) & $6.0(3)$ & $10.7(3)$ & $0(0)$ \\
ODI > 5/hour & $23.1(12 / 49)$ & $37(10 / 27)$ & $9(2 / 22)^{*}$ \\
\hline
\end{tabular}

AHI - Apnoea hypopnoea index; ODI - Oxygen desaturation index. $* \mathrm{p}=0.02$ vs males.

A number of variables were assessed to see if any risk factors for sleep apnoea could be identified within the cohort (see table 3). AHI correlated significantly with age and neck circumference. There was a statistically significant negative correlation between AHI and \% of BNF maximum dose of antipsychotic drug, i.e. higher doses of antipsychotic predicted a lower AHI score. There was a significant interaction between ODI and age, weight, BMI, neck circumference and systolic blood pressure. In keeping with the observation regarding dose of antipsychotic drug and AHI, there was a statis- tical trend towards a negative relationship between antipsychotic dose and ODI.

Many patients described unrefreshing night sleep or daytime fatigue but there was no significant interaction between AHI and ESS or global PSQI score (see figure 1). Therefore an abnormal ESS or PSQI score did not predict sleep disordered breathing. Only one patient with an AHI > 15 had an abnormally elevated ESS. Similarly, mean ESS scores were not significantly different between those with normal or mild SDB $v s$ those with moderatesevere SDB $(5.4 \pm 5.5$ vs $6.7 \pm 2.8, \mathrm{t}=-0.71$, 
Table 3

Correlation of variables with Apnoea Hypopnoea Index and Oxygen Desaturation Index.

Significant correlations are highlighted in bold

\begin{tabular}{lcc} 
& Apnoea Hypopnea Index & Oxygen Desaturation Index \\
\hline Age & $\mathbf{r}=\mathbf{0 . 4 7 4}$ & $\mathbf{r}=\mathbf{0 . 3 6 1}$ \\
& $\mathbf{p}=\mathbf{0 . 0 0 1}$ & $\mathbf{p}=\mathbf{0 . 0 1 1}$ \\
\hline Weight & $\mathrm{r}=0.221$ & $\mathbf{r}=\mathbf{0 . 3 5 4}$ \\
& $\mathrm{p}=0.124$ & $\mathbf{p}=\mathbf{0 . 0 1 3}$ \\
\hline BMI & $\mathrm{r}=0.211$ & $\mathbf{r}=\mathbf{0 . 3 9 0}$ \\
& $\mathrm{p}=0.141$ & $\mathbf{p}=\mathbf{0 . 0 0 6}$ \\
\hline Neck Circumference & $\mathbf{r}=\mathbf{0 . 3 1 1}$ & $\mathbf{r}=\mathbf{0 . 4 1 2}$ \\
& $\mathbf{p}=\mathbf{0 . 0 2 8}$ & $\mathbf{p}=\mathbf{0 . 0 3}$ \\
\hline Systolic blood pressure & $\mathrm{r}=0.279$ & $\mathbf{r}=\mathbf{0 . 3 8 3}$ \\
\hline Diastolic blood pressure & $\mathrm{p}=0.052$ & $\mathbf{p}=\mathbf{0 . 0 0 7}$ \\
\hline \% maximum BNF dose of antipsychotic & $\mathrm{r}=-0.036$ & $\mathrm{r}=0.194$ \\
& $\mathrm{p}=0.805$ & $\mathrm{p}=0.186$ \\
\hline Number of psychotropic drugs & $\mathbf{r}=\mathbf{- 0 . 3 8 2}$ & $\mathrm{r}=-0.307$ \\
& $\mathbf{p}=\mathbf{0 . 0 1 4}$ & $\mathrm{p}=0.054$ \\
\hline ESS & $\mathrm{r}=-0.028$ & $\mathrm{r}=0.095$ \\
& $\mathrm{p}=0.847$ & $\mathrm{p}=0.515$ \\
\hline PSQI & $\mathrm{r}=0.176$ & $\mathrm{r}=0.245$ \\
& $\mathrm{p}=0.220$ & $\mathrm{p}=0.090$ \\
\hline BMI - Body Mass Ins & $\mathrm{r}=0.003$ & $\mathrm{r}=0.155$ \\
& $\mathrm{p}=0.986$ & $\mathrm{p}=0.297$ \\
\hline
\end{tabular}

BMI - Body Mass Index; BNF - British National Formulary; ESS - Epworth Sleepiness Scale; PSQI - Pittsburgh Sleep Questionnaire Inventory.

df $48, \mathrm{p}=0.48)$. ESS had a sensitivity of 0.17 (95\% CI, 0.065-036) and a specificity of 0.81 (95\% CI, 0.57-0.94) for predicting sleep disordered breathing; PSQI had a sensitivity of 0.55 (95\% CI, 036-0.73) and a specificity of 0.37 (95\% CI, 0.17-0.61) for predicting sleep disordered breathing.

Linear regression analysis was performed with AHI as the dependent variable and the independent variables age, gender, neck circumference, BMI, antipsychotic treatment, benzodiazepine treatment and total number of psychotropic drugs entered into the model. The overall model was significant $(\mathrm{F}=$ 3.36, df 7, $p=0.006)$ and $R^{2}=0.36$. Age $(\beta=$ $0.41, \mathrm{p}=0.002)$, gender $(\beta=-0.51, \mathrm{p}=$ $0.027)$ and $\mathrm{BMI}(\beta=0.43, \mathrm{p}=0.015)$ were independent predictors of AHI. Similarly, binary logistic regression with abnormal AHI $(>5)$ as the dependent variable revealed that male gender (OR 21.9, 95\%CI 1.3-370.3, $\mathrm{p}=$ $0.032)$ and $\mathrm{BMI}>25 \mathrm{~kg} / \mathrm{m}^{2}(\mathrm{OR} 36.1,95 \%$ CI 1.3-990.1, $\mathrm{p}=0.034$ ) were independent predictors of sleep-disordered breathing. 


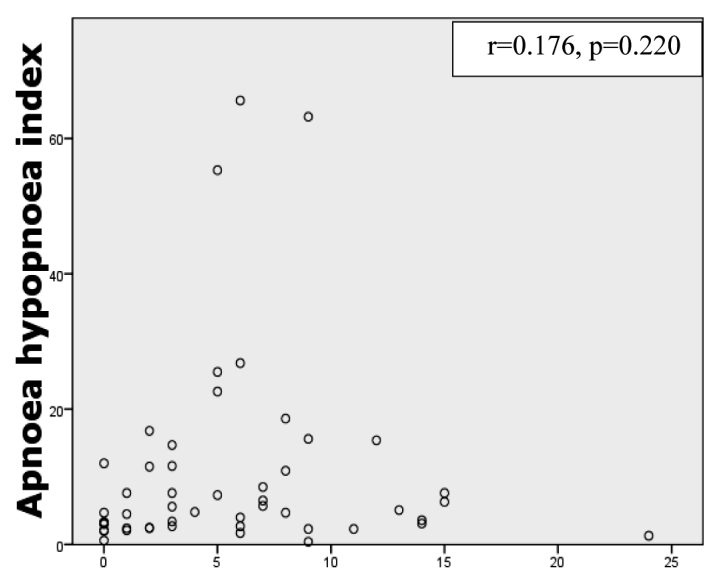

Epworth Sleepiness Scale

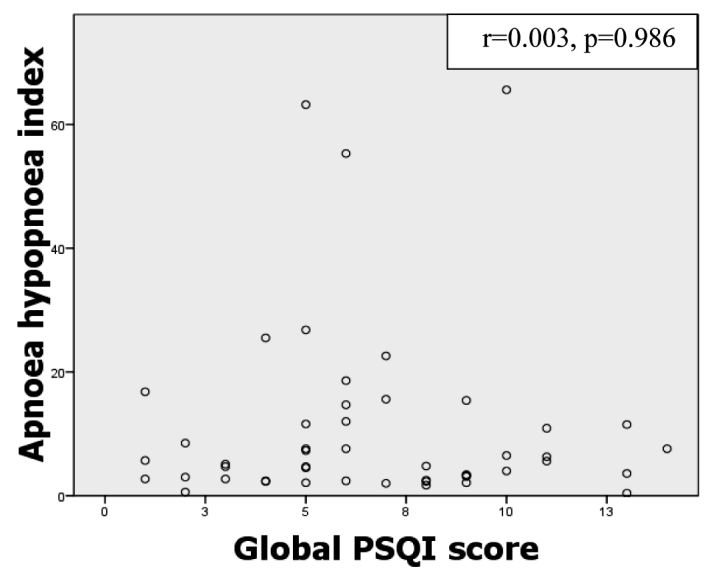

Figure 1. Graphs showing the relationship between AHI and ESS and PSQI scores.

\section{Discussion}

Poor sleep quality correlates inversely with quality of life in psychiatric populations even after correcting for adverse drug effects ${ }^{17,18}$. The true incidence of sleep apnoea and in particular the obstructive sleep apnoea syndrome in those with long term mental health problems maintained on psychotropic medication is unclear with few systematic studies of community cohorts. Obstructive sleep apnoea is treatable and the treatment has been shown to improve overnight sleep quality but also quality of life and cognition in the general population ${ }^{4}$.

In this study of community based psychiatric patients we screened for sleep apnoea with a domiciliary partial polysomnography study and also recorded subjective patient-reported symptoms of sleep disturbance using 
the ESS and PSQI. 52\% of patients had an AHI of $>5$ and $20 \%$ had obstructive sleep apnoea syndrome as defined by an AHI > $15 / \mathrm{hr}$; this prevalence is above that of the background general population. Many large cohort studies have estimated the prevalence of sleep disordered breathing in the normal population and OSA affects $2-4 \%$ of middle aged adults with men affected more than women in all studies. $9-15 \%$ of those between 30 and 60 have an AHI of $>5 / \mathrm{hr}^{3,4}$.

Abnormal ESS $>10$ or PSQI $>5$ did not correlate with a raised AHI although many patients described poor quality sleep. This suggests that these commonly used subjective screening questionnaires may not be useful in this population to detect sleep apnoea although the sample size was small and this would have to be explored further in a larger cohort. More males had sleep apnoea and increasing age and BMI were associated with a higher risk of sleep apnoea, which is similar to findings from other studies.

Previous studies of sleep disturbance in psychiatric disorders demonstrated disrupted sleep architecture but did not look for the presence or absence of sleep apnoea ${ }^{1,19-21}$. Psychotropic medications improve sleep architecture but more recent studies reflect the growing awareness of the negative impact of psychotropic medication on weight gain and their association with metabolic syndrome ${ }^{22}$. Increased body mass index is strongly associated with sleep apnoea in the non-psychiatric population ${ }^{3,4}$. Little has been published on the relationship between sleep apnoea and antipsychotic medication but two case reports exist describing the development of sleep apnoea after initiation of an antipsychotic ${ }^{11,23}$.

There are high rates of depressive symptoms in patients with OSA which improve with OSA treatment ${ }^{24}$. There is also evidence for increased rates of OSA in patients with major depressive disorder with a single overnight home sleep study in 19 patients compared to 15 controls showing increased rates of sleep disordered breathing ${ }^{25}$. These patients were selected without screening for any symptoms of OSA. A more recent study looked at 51 individuals already enrolled in a study of comorbid depression and insomnia with an overnight sleep study and found that $39 \%$ met criteria for OSA, using an AHI cut-off of 15/hr. Those with OSA were more likely to be male and have a higher body mass index ${ }^{26}$. Psychiatric comorbidity in OSA patients was examined in a large retrospective chart review of $>100,000$ OSA patients. A significantly higher prevalence of psychiatric disorders was found in this group compared to the non-OSA population $^{11}$. Within patients referred to a sleep clinic from an inpatient psychiatric hospital who underwent polysomnography, obesity, increasing age, chronic neuroleptic use and male gender were found to be risk factors for sleep apnoea ${ }^{27}$. The author concluded that overweight psychiatric patients and those on chronic neuroleptic treatment should be evaluated for sleep apnoea if signs and symptoms of this disorder are present.

In schizophrenia patients, two studies found no differences in sleep disordered breathing between patient and normal controls $^{5,28}$, however it should be noted that the BMI of patients with schizophrenia was similar to the control subjects in both studies which may cast doubt on how representative these samples were. In contrast an older population (mean age 59 years) studied by Ancoli-Israel ${ }^{6}$ showed that $48 \%$ of the population had an AHI of $>10$ events per hour. The most recent study to look at sleep apnoea in psychiatric outpatients showed that $9.7 \%$ of their population reported a diagnosis of sleep apnoea although sleep studies were not performed ${ }^{7}$. 
One limitation of a number of previous studies is the selection of in-patient populations or those already referred to sleep services. There have been few studies that have screened community populations. Common daytime symptoms of sleep apnoea may be misattributed to the patients' negative symptoms or side effects of psychotropic medication $^{27}$. Additionally many psychiatric patients may not have a bed partner to reliably report snoring or the presence of apnoeas.

Most of our patients had a mixed or predominantly obstructive pattern suggesting that adiposity and weight gain may be the biggest risk factors as in the non-psychiatric population. Sedative medications can cause central sleep apnoeas (pauses in breathing that are not associated with snoring) but only a small percentage of our patients had central apnoeas. There was also no significant interaction between the total number of psychotropic drugs and AHI, suggesting that pharmacological treatment is not mediating the development of central apnoea in this population. The numbers in our study may have been too small to show any significant association as the majority of patients had obstructive sleep apnoea.

With regard to the current study, there are several methodological limitations. The design did not allow for comparisons between drug-naïve patients and those established on psychotropic medication, and we are therefore unable to establish the impact of psychotropic medication on the sleep parameters investigated in this study. The sample size did not differentiate between different psychiatric diagnoses, so we are unable to identify psychiatric diagnostic sub-groups at higher risk of OSA. The lack of a control group prevents any comparison with the background population, although rates of OSA in the background population have been studied in many countries and yielded generally consistent results. Finally, a limited domiciliary sleep study was performed to improve patient compliance but there is no data available relating to sleep architecture or the presence of other sleep disorders such as restless legs syndrome.

In summary, these findings suggest that sleep disordered breathing and OSA is common in community psychiatric patients. The rates were higher than those expected within the background population. The well-established increased prevalence of obesity may be responsible for the relatively high prevalence of sleep apnoea in this population. Increasing age, male gender and elevated BMI were independent risk factors for sleep apnoea in this population, and this group should be particularly monitored for symptoms suggestive of a sleep disorder. The standard questionnaire screening tools for assessment in the clinic may not be suitable for this population. Given that OSA represents a potentially treatable cause of symptoms that can contribute to daytime sleepiness and impaired quality of life, further larger scale prospective studies are needed to investigate the prevalence of obstructive sleep apnoea syndrome and response to treatment.

\section{Acknowledgements}

The study was sponsored by the Nortumbesland Tyne and Wear NHS Trust and Dr Mackin holds a Department of Health Clinician Scientist Fellowship.

\section{References}

1. Benca RM, Obermeyer WH, Thisted RA, Gillin JC. Sleep and psychiatric disorders. A meta-analysis. Arch Gen Psychiatry 1992; 49: 651-668; discussion 669-70. 
2. Cohrs S. Sleep disturbances in patients with schizophrenia: impact and effect of antipsychotics. CNS Drugs 2008; 22: 939-962.

3. Young T, Palta M, Dempsey J, Skatrud J, Weber S, Badr S. The occurrence of sleep-disordered breathing among middle-aged adults. N Engl J Med 1993; 328: 1230-1235.

4. Ferini-Strambi L, Fantini ML, Castronovo C. Epidemiology of obstructive sleep apnea syndrome. Minerva Med 2004; 95: 187-202.

5. Takahashi KI, Shimizu T, Sugita T, Saito Y, Takahashi Y, Hishikawa Y. Prevalence of sleep-related respiratory disorders in 101 schizophrenic inpatients. Psychiatry Clin Neurosci 1998; 52: 229-231.

6. Ancoli-Israel S, Martin J, Jones DW, Caligiuri M, Patterson T, Harris MJ, et al. Sleep-disordered breathing and periodic limb movements in sleep in older patients with schizophrenia. Biol Psychiatry 1999; 45: 1426-1432.

7. Nasr S, Wendt B, Kora S. Increased incidence of sleep apnea in psychiatric outpatients. Ann Clin Psychiatry; 22: 29-32.

8. Mackin P, Bishop D, Watkinson H, Gallagher P, Ferrier IN. Metabolic disease and cardiovascular risk in people treated with antipsychotics in the community. Br J Psychiatry 2007; 191: 23-29.

9. Vgontzas AN, Bixler EO, Chrousos GP. Sleep apnea is a manifestation of the metabolic syndrome. Sleep Med Rev 2005; 9: 211-224.

10. Vgontzas AN. Does obesity play a major role in the pathogenesis of sleep apnoea and its associated manifestations via inflammation, visceral adiposity, and insulin resistance? Arch Physiol Biochem 2008; 114: 211-223.

11. Sharafkhaneh A, Giray N, Richardson P, Young T, Hirshkowitz M. Association of psychiatric disorders and sleep apnea in a large cohort. Sleep 2005; 28: 1405-1411.

12. Boufidis S, Kosmidis MH, Bozikas VP, Daskalopoulou-Vlahoyianni E, Pitsavas S, Karavatos A. Treatment outcome of obstructive sleep apnea syndrome in a patient with schizophrenia: case report. Int J Psychiatry Med 2003; 33: 305-310.

13. Johns MW. A new method for measuring daytime sleepiness: the Epworth sleepiness scale. Sleep 1991; 14: 540-545.

14. Buysse DJ, Reynolds CF, 3rd, Monk TH, Berman SR, Kupfer DJ. The Pittsburgh Sleep Quality Index: a new instrument for psychiatric practice and research. Psychiatry Res 1989; 28: 193-213.

15. Ng SS, Chan TO, To KW, Ngai J, Tung A, Ko FW, et al. Validation of Embletta portable diagnostic system for identifying patients with suspected obstructive sleep apnoea syndrome (OSAS). Respirology 2010; 15: 336-342.
16. Kushida CA, Littner MR, Morgenthaler T, Alessi CA, Bailey D, Coleman J, Jr., et al. Practice parameters for the indications for polysomnography and related procedures: an update for 2005. Sleep 2005; 28: 499-521.

17. Doi Y, Minowa M, Uchiyama M, Okawa M. Subjective sleep quality and sleep problems in the general Japanese adult population. Psychiatry Clin Neurosci 2001; 55: 213-215.

18. Ritsner M, Kurs R, Ponizovsky A, Hadjez J. Perceived quality of life in schizophrenia: relationships to sleep quality. Qual Life Res 2004; 13: 783-791.

19. Chouinard S, Poulin J, Stip E, Godbout R. Sleep in untreated patients with schizophrenia: a meta-analysis. Schizophr Bull 2004; 30: 957-967.

20. Gaillard JM. Biochemical pharmacology of paradoxical sleep. Br J Clin Pharmacol 1983; 16 Suppl 2: 205S-230S.

21. Kempenaers C, Kerkhofs M, Linkowski P, Mendlewicz J. Sleep EEG variables in young schizophrenic and depressive patients. Biol Psychiatry 1988; 24: 833-838.

22. Reynolds GP, Kirk SL. Metabolic side effects of antipsychotic drug treatment-pharmacological mechanisms. Pharmacol Ther 2010; 125: 169-179.

23. Wirshing DA, Pierre JM, Wirshing WC. Sleep apnea associated with antipsychotic-induced obesity. J Clin Psychiatry 2002; 63: 369-370.

24. Millman RP, Fogel BS, McNamara ME, Carlisle CC. Depression as a manifestation of obstructive sleep apnea: reversal with nasal continuous positive airway pressure. J Clin Psychiatry 1989; 50: 348-351.

25. Deldin PJ, Phillips LK, Thomas RJ. A preliminary study of sleep-disordered breathing in major depressive disorder. Sleep Med 2006; 7: 131-139.

26. Ong JC, Gress JL, San Pedro-Salcedo MG, Manber R. Frequency and predictors of obstructive sleep apnea among individuals with major depressive disorder and insomnia. J Psychosom Res 2009; 67: 135-141.

27. Winkelman JW. Schizophrenia, obesity, and obstructive sleep apnea. J Clin Psychiatry 2001; 62: 8-11.

28. Benson KL, Zarcone VP. Schizophrenia. In: Kryger MH, Roth T, Dement WC, editors. Principles and practice of sleep medicine. 3rd ed. Philadelphia: Saunders; 2000. p. 1159-1167.

Author for correspondence:

Dr. Kirstie N Anderson

Department of Neurology, Royal Victoria Infirmary

Queen Victoria Road, Newcastle upon Tyne

NE1 4LP

Phone: 01912823833

Fax: 01912823435

E-mail: kirstie.anderson@nuth.nhs.uk 